\title{
NATIONALISM AND RASHTRIYA SWAYAMSEVAK SANGH IN THE POST-INDEPENDENCE PERIOD
} स्वातंत्र्योत्तर काल में राष्ट्रवाद एवम् राष्ट्रिय स्वंय सेवक संघ

\author{
Dr. Preeti Pandey ${ }^{* 1} 凹$ \\ ${ }^{* 1}$ Guest Lecturer, School of Ancient Indian History, Culture and Archeology, Vikram University \\ Ujjain, India
}

DOI: https://doi.org/10.29121/granthaalayah.v9.i6.2021.4050

Article Type: Research Article

Article Citation: Dr. Preeti Pandey. (2021). NATIONALISM AND RASHTRIYA SWAYAMSEVAK SANGH IN THE POSTINDEPENDENCE PERIOD. International Journal of Research -GRANTHAALAYAH, 9(6), 401 - 404.

https://doi.org/10.29121/granthaalayah.v9.i6.2021.4050

Received Date: 15 June 2021

Accepted Date: 30 June 2021

Keywords: स्वातंत्र्योत्तर, राष्ट्रवाद, सेवक

\section{ABSTRACT}

English: Nationalism is a subject in which there has been controversy from time to time. When it comes to Rashtriya Swayamsevak Sangh, there are often misconceptions that it opposes nationalism, but this is a myth. For this purpose, this letter has been written with special reference to the post-independence period. Writing this letter is very relevant at present because even today many people do not know the true nature of this organization and are keeping distance due to this. We find that if understood in the context of proper evidence, then this organization is also a socio-cultural organization like other organizations, which since its inception in 1925 till today is determined towards social upliftment and national upliftment. Discipline is its main weapon. It means that the Rashtriya Swayamsevak Sangh cooperated with the government in resolving the problems that arose after independence, although it was banned but again proved to be immaculate and moved forward in the forward responsibility with great energy. For this research, The subject has been reviewed in the then circumstances by studying books, newspapers related to Golwalkar ji and related to nationalism.

Hindi: स्वातंत्र्योत्तर काल में राष्ट्रवाद की भावना पर प्रायः प्रश्नचिन्ह लगाये जाते रहे हैं क्योंकि सबका मानना है कि स्वतंत्रता प्राप्त होते ही राष्ट्रवाद की आवश्यकता की पूर्ति हो जाती है किन्तु यह एक भ्रामक चिन्तन है। राष्ट्र के प्रति, प्रेम, विश्वास, आस्था एवं स्वार्थ के त्याग का संयुक्त भाव है जिसका सम्बन्ध राष्ट्र के अस्तित्व से होता है। जब स्वतंत्रता आन्दोलन चल रहा था तब यह अवधारणा एक सामान्य भाव से सबके बीच में पनप रही थी एवं आवेग तथा संवेग अपने चरम पर था। उत्तर काल में इसकी गति शनैः अवश्य हो जाती है किन्तु राष्ट्रवाद की भावना का अन्त नहीं होता। इस काल में यह अवधारणा कई धाराओं में विकसित होती दिखती है। इसी में एक धारा का विकास 1925 में जन्मे संगठन राष्ट्रीय स्वंय सेवक संघ में पोषित होती है।

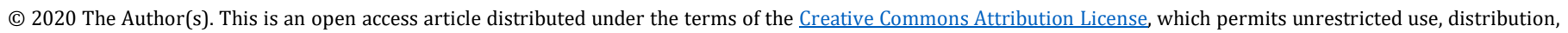
and reproduction in any medium, provided the original author and source are credited. 
स्वातंत्र्योत्तर काल में राष्ट्रवाद एवम् राष्ट्रीय स्वंय सेवक संघ

\section{1. प्रस्तावना}

स्वातंत्र्योत्तर काल में राष्ट्रवाद की भावना पर प्रायः प्रश्नचिन्ह लगाये जाते रहे हैं क्योंकि सबका मानना है कि स्वतंत्रता प्राप्त होते ही राष्ट्रवाद की आवश्यकता की पूर्ति हो जाती है किन्तु यह एक भ्रामक चिन्तन है। राष्ट्र के प्रति, प्रेम, विश्वास, आस्था एवं स्वार्थ के त्याग का संयुक्त भाव है जिसका सम्बन्ध राष्ट्र के अस्तित्व से होता है। जब स्वतंत्रता आन्दोलन चल रहा था तब यह अवधारणा एक सामान्य भाव से सबके बीच में पनप रही थी एवं आवेग तथा संवेग अपने चरम पर था। उत्तर काल में इसकी गति शनैः अवश्य हो जाती है किन्तु राष्ट्रवाद की भावना का अन्त नहीं होता। इस काल में यह अवधारणा कई धाराओं में विकसित होती दिखती है। इसी में एक धारा का विकास 1925 में जन्मे संगठन राष्ट्रीय स्वंय सेवक संघ में पोषित होती है।

इस पत्र में 1947 के बाद राष्ट्रवाद की उसी धारा वैश्लेषिक अध्ययन स्वातंत्र्योत्तर काल में जायेगा। हम देखेंगे कि इस कालखण्ड में राष्ट्रवाद का विकास राष्ट्रीय स्वंय सेवक संघ में किस प्रकार किया जा रहा था। तथा इसका भारतीय राष्ट्रवाद की अन्य धाराओं पर क्या प्रभाव पड़ रहा था।

15 अगस्त 1947 में जैसे ही भारत को स्वतंत्रता मिली कई प्रकार की विभीषिकाओं ने भी जन्म ले लिया ऐसा लग रहा था कि स्वतंत्रता न मिली हो कोई आपदा आ गई हो। विभाजन दंगे देशी रियासतों का विद्रोही स्वरूप तथा सर्वोपरि गांधीहत्या विभिन्न राजनीतिक, सामाजिक, समस्याओं को लेकर आ गई। ऐसे में संघ की भूमिका एवं उनकी राष्ट्रवादी हष्टिकोण दृष्टव्य होगा।

स्वतंत्रता काल में राष्ट्रीय स्वंय सेवा संघ का नेतृत्व माधवराव सदाशिव राव गोलवलकर 'गुरूजी' कर रहे थे जो कि संघ के द्वितीय सरसंघ चालाक थे। इन विषय परिस्थितियों में उन्होनें राष्ट्र संघ के हृ्टिकोण को पूर्णरूप से राष्ट्रवाद बना कर रखा।

1947 को विभाजन आधारित स्वतंत्रता बहुत सुखद घटनाओं का संचार नहीं कर रही थी। पूरे भारत में कटुता के बीज पनप गये थे जो आसानी से समाप्त नहीं हो सकते थे। इस माहौल में यद्यपि कांग्रेस को अन्य नेता प्रसत्न थे किन्तु गाँधीजी पूर्ण रूप से सम्मिलित नहीं थे और उन्होनें कहा था कि मेरा भ्रम टूट गया है भले ही आपना न टूटा हो। [1]

स्वतंत्रता के ऐसे हिंसक स्वरूप की कल्पना किसी ने नहीं की थी। रावलपिण्डी, अमृतसर, लाहौर आदि स्थानों पर हिन्दुओं पर भरपूर अत्याचार हो रहे थे। जगह-जगह संघ के नेतृत्व में सुरक्षादल खड़े किये गये। गुरूजी ने पाकिस्तान के इन सभी स्थानों में संघ के अधिकारियों को डटकर कार्य करने के निर्देश दिये। विभाजन में इस कालखण्ड पर पुस्तकें लिखने वाले निरपेक्ष लोगों ने स्वंयसेवको की वीरता, पराक्रम एवं समाज की रक्षा में प्राणोत्सर्ग करने की प्रशंसा की है। प्रो.ए.एन.बाली की पुस्तक में उन्होनें स्पष्ट किया कि रा.स्व.से.संघ के स्वंय सेवकों ने रात-रात भर पहरा देकर हिन्दू एवं सिख लोगों को दंगाईयों से बचाया। और इस कार्य में तो कई वीरगति भी प्राप्त कर गये। [2] गुरूजी ने इन कार्यों को समाज के रक्षार्थ निभाया गया आवश्यक कर्त्तव्य माना और इसी कारण कोई श्रेय लेने का प्रयत्न नहीं किया। गुरूजी विभाजन को अन्तिम सत्य नहीं मानते थे। अखण्ड मातृभूमि का स्वप्र वे सदा ही हृदय मे पालते रहे।

विभाजन के बाद देशी रियासतों का एकीकरण एक बड़ी समस्या थी जिसमें कश्मीर की समस्या अत्यन्त विकट थी। राजा हरीसिंह जो स्वंय हिन्दू थे वे मुस्लिम बहुत काश्मीर को किसी भी शर्त में पाकिस्तान के साथ मिलाने देना नहीं चाहते थे। राष्ट्रीय स्वंय सेवक संघ के प्रान्त संघचालक पं. प्रेमनाथ डोंगरा ने महाराज से निजी चर्चा की एवं अनुरोध किया कि वे भारत सरकार में विलय हो जाये। [3]

गोलवलकर जी महराजा हरीसिंह के विश्वासपात्र थे। [4] भारत सरकार के सरदार पटेल ने गुरूजी को मध्यस्थ बनाकर उन्हें 17 अक्टूबर 1947 को गुरूजी ने श्रीनगर जाकर महाराज से बात की एवं उन्हें विलय के लिये तैयार किया। [5] शीघ्र ही भारत सरकार के प्रयासों से कश्मीर भारत का अंग बना।

इसी प्रकार गोवा स्वाधीनता संग्राम मे पुणे के संघचालक विनायक राव आप्टे ने महत्वपूर्ण भूमिका निभाई। अनेक प्रमुख संघ कर्ताओं ने महत्वपूर्ण गुरिल्ला रणनीति अपनाई और गोवा की मुक्ति में कई स्वंयसेवकों के बलिदान के बाद अन्त में वह भी स्वतंत्र हो गया। उज्जैन के राजा भाऊ महाकाल मध्यभारत से जत्थे का नेतृत्व कर रहे थे और इसमें शहीद हो गये। इस प्रकार देशी रियासतों के एकीकरण में संघ ने भारत सरकार का पूर्ण सहयोग दिया।

किन्तु इसी काल में अविश्वास एवं संदेह का वातावरण भी बना दिया था। देश की सरकार को संचालित करने वाली कांग्रेस दल प्रारंभ से ही राष्ट्रीय स्वंय सेवक संघ को विरोधी संस्था मानती थी। गोलवलकर जी के नेतृध̈व में संघ के विकासवादी स्वरूप एवं जनमानस में उनके प्रति बढ़ती लोकप्रियता ने सरकार को भयभीत कर दिया और वह संघ की तुलना विद्रोही एवं 
प्रतिक्रियावादी पार्टी मुस्लिम लीग से करने लगी सरकारी कांग्रेस दल ऐसा मौका ढूँढने लगी जिससे इस संस्था पर अंकुश लगाया जा सके। शीघ्र ही महात्मा गांधी की हत्या से उन्हें ये अवसर भी मिल गया। [6]

विभाजन के विप्लव में महात्मा गांधी को बलिदान देना पड़ा। गांधीजी की मुस्लिम तुष्टीकरण की नीति ने हिन्दुओं को उपेक्षित अनुभव करने पर बाध्य कर दिया, फलस्वरूप प्रतिक्रिया होना स्वाभाविक था। 30 जनवरी 1948 को नाथुराम गोडसे नामक हिन्दूवादी व्यक्ति ने उनकी गोली मार हत्या कर दी। यह निश्चित रूप से एक निन्दनीय कृत्य था जिसकी भरपाई नहीं की जा सकती। महात्मा गांधी की हत्या पूरे राष्ट्र के लिये शोक का विषय था। भारत सरकार ने इस हत्या काण्ड के आधार पर राष्ट्रीय स्वंय सेवक संघ पर प्रतिबन्ध लगा दिया गया। भारत सरकार ने विज्ञात् के द्वारा स्पष्ट किया कि राष्ट्र के लिये खतरा होने के कारण राष्ट्रीय स्वंय सेवक संघ एक अवैध संगठन है। जो कि लूट हत्या एवं गोला बारूद जैसे कृत्यों में लगी है।

इस प्रतिबन्ध की प्रतिक्रिया पर गुरूजी ने अपना वक्तव्य दिया जो पाकिस्तान के डॉन सामाचार पत्र में हत्या बाद में अन्य पत्रों में इसके अनुसार-

"राष्ट्रीय स्वंय सेवक संघ की प्रारंभ से ही यह नीति रही है कि सरकारी प्रतिबन्धों का पालना करते हुये ही अपने कार्य किये जाये। इस समय सरकार ने उसे अवैध घोषित कर दिया है। अतः ने यही उचित समझता हूँ कि प्रतिबन्ध हटाये जाने तक संघ को विसर्जित करता हूँ। तथापि सरकार ने संघ पर जो आरोप लगाये हैं उन्हें मैं पूर्णतया अस्वीकार करता हूँ। स्वयं विनोबा भावे ने भी इस तथ्य को नकारा था कि राष्ट्रीय स्वंय सेवक संघ पर गांधी हत्या का दोष मढ़ना भूल है।" [7]

4 फरवरी 1948 का लगा संघ एवं गुरूजी पर प्रतिबन्ध 13 जुलाई 1949 को हटाया गया जस्टिस कपूर आयोग ने गुरूजी एवं राष्ट्रीय स्वंय सेवक संघ को इस प्रकरण में संलिप्त होने की संभावना को पूर्ण रूप से खारिज कर दिया। प्रतिबन्ध के 18 मास में संघ के स्वरूप को विवादित एवं अतिवादी गढ़ने की चेष्टा की गई किन्तु यह कुचेष्टा असफल रही और राष्ट्रीय स्वंय सेवक संघ पुनः अपने स्वरूप में वापस आने लगा। गुरूजी ने कहा कि पुराना सब भूल कर हमें मातृभूमि की उत्रति के स्वप्र को साकार करना है। [8]

प्रतिबंध के बाद सब में अपार भीड़ जुटने लगी और जनता स्वंय सत्य असत्य का भेद करने लगी। आगामी 24 वर्षों में गुरूजी ने संघ को अत्यन्त महत्वपूर्ण स्वरूप प्रदान किया। सर्वांगीण राष्ट्रीय पुनुरूत्थान को लक्ष्य लेकर संगठन में तानाशाही जैसी कोई व्यवस्था नहीं है। समाज के हर क्षेत्र में विभित्र अनुषांगिक संगठन खोलकर संघ ने समाज की विभित्र रूपों में सेवा की।

राष्ट्र के स्वरूप को गुरूजी ने बहुत सहज शब्दों में स्पष्ट किया था। उनका मानना था कि समान भूमि, समान हित, समान भाव एवं राष्ट्र के लिये समान सम्मान एवं राष्ट्र की नींव डालता है। इस प्रकार भौगोलिक क्षेत्र राष्ट्र एवं उसके प्रति आस्था की भावना राष्ट्रीयता कहलाती है। [9]

कुछ लोगों का मानना था कि अंग्रेजों से विरोध और आन्दोलन ही राष्ट्र कार्य है किन्तु मात्र नकारात्मक रीति से राष्ट्र प्रेम नहीं उत्पन्न होता है। विदेशी सत्यधीश मानते थे कि राष्ट्र भावना भारत में पहले थी ही नहीं इसी कारण से । A new nation In the making और । A new nation born जैसी पंक्तियां कहीं गई। भारत को वो नवोदित राष्ट्र मानते थे।

संघ ने स्पष्ट किया कि जब अंग्रेज एवं मुस्लिम भारत में नहीं आये थे तब भी वह एक राष्ट्र था। अथर्ववेद में जब यह उद्घोषणा हुई कि पृथ्वी हमारी माता है और हम उसके पुत्र है। उसी समय राष्ट्रीयता का उद्घोष हो गया था। राष्ट्र की मुख्य धारा में छोटी-छोटी अनेक नदियों मिलती रही और भारतीय संस्कृति उसे आत्मसात् करती रही।

निश्चित रूप से राष्ट्र मनुष्यों का एक निष्चित भूमि में समुच्चय मात्र नहीं है वरन् एक विशिष्ट जीवन पद्धति है जिसमें जीवन में आदर्श, संस्कृति, अनूभूतियों, भावनाओं, विश्वास, परंपराओं आदि का सम्मिलित रूप हो। हम जब सभी लोगों की आचारों विचारों से एकाकार हो जाते है। तो इस सुव्यवस्थित सुसंगठिsत समाज जिस विशिष्ट प्रदेश में निवास करता है। उसे राष्ट्र एवं उसके प्रति आस्था राष्ट्रवाद कहलाती है। [10]

निश्चित रूप से स्वातंत्र्योत्तर काल में राष्ट्रवाद का स्वरूप विच्छूखलित हो गया था और उसकी धारा अलग रूप में विकसित हो रही थी किन्तु ऊर्जा भांति उसने मात्र अपना स्वरूप बदला था विनष्ट नहीं हुई थी। राष्ट्रीय स्वंय सेवक संघ के रूप में राष्ट्रवाद की एक धारा पनपी और विकसित हुई। [11]

यद्यपि इस संगठन को विवादित स्वरूप में देखा गया किन्तु इसमें संदेह नहीं है कि राष्ट्रीय स्वंय सेवक संघ ने अपने राष्ट्र के प्रति कर्त्तव्य को नहीं छोड़ा इसी का परिणाम है कि उत्तरोतराकर इस संगठन ने अपना विकास किया और जनमानस में अपनी जगह बनाई। 


\section{SOURCES OF FUNDING}

None.

\section{CONFLICT OF INTEREST}

None.

\section{ACKNOWLEDGMENT}

None.

\section{REFERENCES}

[1] चं.प. भिशीकर नवयुग प्रवर्तक गुरूजी पृ.12

[2] एच.पी. हटसन द ग्रेट डिवाइड पृ. 236

[3] राष्ट्रीय स्वंय सेवक संघ के सरसंघचालक के रूप में गुरूजी; प्रीति पाण्डे पृ.281

[4] पूर्ववत्; पृ.86

[5] मा.स. गोलवलकर; समग्रदर्शन भाग-2 पृ. 10

[6] पूर्ववत् पृ.10

[7] एस.एम.चाँद; महात्मा गांधी और साम्प्रदायिक एकता पृ. 108

[8] विजयकुमार; अपने परम पुज्यनीय सरसंघचालक पृ.22

[9] मा.स. गोलवलकर; राष्ट्र पृ.156

[10] श्री गुरूजी जीवन प्रसंग भाग-2 पृ.135

[11] मा.स.गोलवलकर; राष्ट्र पृ.164 